turbances, investigation, and diagnosis are straightforward, if somewhat bland, accounts of largely well established information. My impression is that the authors are at their best in describing the neurological, and particularly the malignant, complications of coeliac disease, which is a subject for which the Birmingham school will be most remembered.

It is in the other chapters on mucosal and cellular abnormalities, immunology, gluten challenge, and aetiology where difficulties arise, but this is understandable since these are subjects that continue to evoke controversy and claim and counter claim. There is much overlap among these chapters, and it might have been more useful to condense those superfluous parts into one expanded chapter on pathogenesis, including gluten challenge: the value of gluten challenge lies more in investigative research than everyday clinical diagnosis.

Also in these sections the authors have allowed themselves to be led astray by quoting too many opinions of writers whose work they extensively review, rather than imposing more of their own thoughts and discarding material that no longer commands respect or credibility. As it stands, the text often fails to move incisively onwards and to impel the reader with it; instead we meander up every nook and cranny, the resulting perspective being always cautious and somewhat retrospective. Chapter 3, for example, contains 326 references on mucosal injury yet less than a quarter are of work published since 1975 and less than 5\% later than 1980 .

Again the reader is given an exhaustive, or maybe exhausting, recital of every imaginable immunological mechanism ever proferred to explain the mucosal lesion in coeliac disease but from which no coherent view of pathogenesis arises to help guide the less familiar reader through this difficult terrain. It was also difficult to perceive what the authors themselves believe about the nature of epithelial damage, or so called "lymphocytic infiltration" of the epithelial cell layer. Do "high numbers of lymphocytes within the epithelium" reflect the primary event resulting in the classic "flat" mucosa, or is the latter secondary to events occurring solely in the lamina propria? And, if so, is lymphoid infiltration of epithelium then just another irrelevant epiphenomenon? In these particular aspects of the book I found little to catch the eye or quicken the pulse.

Nevertheless, this handsomely bound book invites perusal and is certainly good value for money. Apart from becoming useful library stock, it should also find its way into many personal collections since there is much in its 281 pages to satisfy the needs of "gastroenterologists and others who may encounter the condition in their practice and research."

Coeliac Disease. W T Cooke and G K T Holmes. (Pp 281; £30.) Churchill Livingstone. 1983.

\title{
Vulnerable minorities
}

\section{J A BLACK}

This issue of Radical Community Medicine is devoted to "Race and Health"; most of the articles deal with the politics and social aspects of racial discrimination as it affects health care, while two authors discuss racialism within the organisation of the National Health Service.

Regrettably, the standard of the contributions, with a few exceptions, is mediocre, and their meaning often obscured by sociological jargon. Understandably, given the way in which British society is permeated by racial discrimination, some of the authors have a somewhat paranoid viewpoint. To take the stance that ethnic minorities receive a second class standard of medical care, and at the same time to decry as another form of discrimination attempts to study what has gone wrong, is destructive. In reading the journal one gets the impression that the only groups with any problems are the black or brown; this misrepresentation inhibits a proper consideration of the wider aspects of the subject and has had a distorting and stultifying effect on a number of recent meetings of organisations concerned with the health of ethnic minorities.

Clearly, doctors-family doctors in particular-and health workers in general need to be better informed about sickle cell disease, thalassaemia, and glucose-6-phosphate dehydrogenase deficiency. Many health workers are still unaware that there are clinics for sickle cell disease and thalassaemia, where specialist treatment and genetic counselling are available, nor do they know about such organisations as the Sickle Cell Society, Organisation for Sickle Cell Anaemia Research (Oscar), and the Thalassaemia Society. These facilities are, however, available only in our large cities, and in areas with few families from ethnic minority groups treatment of these potentially disabling conditions is often very poor.

It is not enough, however, to know about the haemoglobinopathies: each ethnic group has distinct problems. For example, it is both insensitive and medically unsound not to recognise that the various Asian groups have different religious and cultural beliefs, and different dietary customs, and that any of these may sometimes affect their health. Iron deficiency anaemia, rickets, and folate deficiency are more likely to occur in a strictly vegetarian Hindu family, whereas there is no such risk among the Sikhs. Linguistic difficulties can cause misunderstandings, anxiety, and inadvertent non-compliance in treatment. Many hospitals make no attempt to train their staff to deal with language problems, or Asian naming systems, or to supply suitable food such as Halal meat for Muslim patients. In one of the few constructive articles in Radical Community Medicine Fedelma Winkler, secretary of the Hackney Community Health Council, describes an imaginative and practical scheme for helping women in Mother's Hospital in Hackney. Several "local women" from the main ethnic minority groups in the borough work in the antenatal clinics and in the wards and are able to "speak for" those whose English is poor or non-existent; they help the medical, nursing, and catering staff, and advise the mothers about the facilities available to them and encourage them in breast feeding and other matters that require an appreciation of the various cultural beliefs in each group.

The contributors to Radical Community Medicine are, I think, telling us that it is not enough to talk about sickle cell anaemia or dietary deficiencies but that we should try to change a system that at present ensures that the ethnic minority family gets the worst housing (Hackney again, though one suspects that other boroughs are just as bad but have not been studied by the Commission for Racial Equality), lowest paid jobs, poorest promotion prospects, and the greatest probability that their school leavers will be unemployed. Illnesses that result from such adverse circumstances are not in fact determined by skin colour or religious belief. Unfortunately, our society is bent on making the rich richer and the poor poorer and the main sufferers, in addition to the usual vulnerable groups, are the ethnic minorities.

Radical Community Medicine. Quarterly journal. Ed Alex ScottSamuel. Winter issue: 1983-4. "Race and Health." (£1·50.) Radical Community Medicine. 1983. 